\title{
Computation Interval-Valued Reliability of Sliding Window System
}

\author{
Akshay Kumar \\ Department of Applied Sciences \\ Tula's Institute, The Engineering and Management College, Dehradun, Uttarakhand, India \\ Corresponding author: akshaykr1001@gmail.com \\ Mangey Ram \\ Department of Mathematics, Computer Science and Engineering \\ Graphic Era Deemed to be University, Dehradun, Uttarakhand, India \\ E-mail: drmrswami@yahoo.com
}

(Received June 28, 2018; Accepted October 2, 2018)

\begin{abstract}
In this study, a sliding window system is revisited and evaluated interval-valued reliability in case of upper and lower form with the help of universal generating function technique and using an algorithm, how to compute the reliability of sliding window system. Computation of probability by interval-valued is most uses but universal generating function method given improved results within it. An example is also taken at the end to demonstrate the system.
\end{abstract}

Keywords- Reliability, Sliding window system, Interval valued, Universal generating function.

\section{Introduction}

Reliability is of utmost importance in human life and plays a key role in engineering systems. In recent years, engineers and mathematicians of several countries have investigated new research in reliability theory. The reliability theory became a focal point as far as studies of systems are concerned because of automations complexity and sensitivity of modern day engineering systems. Ushakov $(1986,1994)$ discussed reliability engineering that plays a key role in real life. The author reviewed and discussed the system reliability and applied it in engineering systems also introduced some basic techniques which are applicable in cutting-edge results, covers probabilistic reliability and statistical reliability etc., presented various techniques \& applications of reliability theory in real life systems. Levitin (2001) considered a redundancy optimization system for a multi-state system, which has a fixed amount of resource for its work performance and resource generator from the subsystem. The suggested algorithm evaluated the optimal system structure and system availability. The system productivity, availability and cost were evaluated from performance based on each element. The main goal of the study was to minimize the cost investment, total demand and to present the demand curve on the basis of system probability. Genetic algorithm was used to solve the problem based on universal generating function (UGF), to compute the system availability, optimal structure function while working element of the subsystem had a maximum performance rate under given demand distribution. Levitin (2002) proposed a linear multi-state sliding window system, which generalized the multistate consecutive $k$-out-of- $r$-from- $n: \mathrm{F}$ system. The considered system consists of $n$ linearly ordered multi-state elements. Each element could have two states: total failure or completely working. In that work, the author discussed that system fails if the total sum of the $r$ consecutive elements performance rate is lower than total allocated weight. Also evaluated various characteristics of the linear multi-state sliding window system with the suggested algorithm to 
International Journal of Mathematical, Engineering and Management Sciences

Vol. 4, No. 1, 108-115, 2019

https://dx.doi.org/10.33889/IJMEMS.2019.4.1-010

find the order of elements and maximum system reliability. Levitin (2003a) introduced a two state linear multi-state sliding window system, which consists of $n$ linearly ordered multi-state elements. The system performance rate was based on given performance weight. The system fails if the quantity of the performance rates is less than total demand. The author presented an algorithm for calculating the reliability of a sliding window system (SWS) to the common supply failures (CSF) and common supply groups (CSG). In that system it also described a method for comparing optimal element distributions of the CSG system reliability. The proposed study computed optimization result with the help of UGF technique and Genetic algorithm. Levitin (2003b) proposed multi-state a system that generalized the consecutive $k$-out-of- $r$-from- $n: F$ system. The considered linear multi-state sliding window system consisted of $n$ ordered multistate elements and each element could have two states. In this study author evaluated the system reliability, mean time to failure (MTTF) and cost of the proposed system using extended universal moment generating function. Levitin (2005) reviewed and studied that the binary system can have two possible states either working or failed but the multi-state system (MSS) has various working (performance states) and many failure modes. The author discussed the binary and multi-state system reliability estimation, optimization and stated the applications in various engineering fields on the basis of the UGF technique.

Utkin and Kozine (2005) considered a non-repairable system and obtained interval-valued reliability and its factors. Gennat and Tibken (2008) analysed the sensitivity of the proposed model using interval arithmetic and computed inner and outer approximation for the desired set of possible measures. Ram and Singh (2010) studied a complex system to characterize system reliability in terms of probability, MTTF, and expected cost. They defined two types of repair, general, and exponential. The proposed system studied incorporating "preemptive-repeat repair discipline" and applying Gumbel-Hougaard family copula, supplementary variable technique and Laplace transformation to evaluate the transition state probabilities and MTTF. Li et al. (2011) considered an MSS and computed the interval-valued reliability, state probabilities of multi-state elements and the precise values of the state performance. They proposed method to determine the reliability of MSS when the available data of elements is insufficient. Authors analyzed the interval-valued state probabilities of elements based on the Bayesian approach and the imprecise Dirichlet model. An UGF algorithm is proposed for obtaining the interval-valued reliability of MSS. Destercke and Sallak (2013) evaluated reliability of MSS with epistemic uncertainties using UGF technique. They discussed various solutions and techniques to compute MSS reliability measures. In this work, the authors provided extension of the UGF method incorporating epistemic uncertainties. They compared the extension with UGF technique on the basis of interval arithmetic operations performed on interval-valued probabilities. Guo et al. (2013) described a new method to determine the system reliability of a machine element when data of the probability density functions of stress and strength are available. Researchers introduced UGF and discussed the discrete interval-value for random variable. From two discrete interval-valued random variables, they computed the experimental data of stress and strength. For computing the upper and lower bounds of reliability the considered stress-strength interference system have been computed using UGF and probability density functions. Ram (2013) studied a survey on system reliability approaches. In the recent year reliability theory becomes the most searching and testing theory. Researchers have proposed various theories and methods of reliability evaluation such as; Bayes approach, coherent systems approach, copula approach, coverage factor approach, designed experiments, distributed system, genetic algorithm, nano and network system approach, etc. in various fields of engineering and physical sciences. Chachi and Hesamian (2015) presented various methods in reliability theory assuming that all measures of 
lifetime density function are definite. They observed that many time measures cannot predict errors in a machine during an experiment and one's judgment in unexpected situations. Authors also discussed interval-valued measures using random variable for coherent system and computed the system reliability. Their study also included an idea of cumulative distribution function and probability density function of the random variable on the basis of interval-valued. Wang et al. (2016) presented interval analysis for cold-standby system and evaluated reliability optimization under parameter uncertainty. They discussed properties of statistical parameters to compute probabilistic properties and used interval value to denote the system optimization problems. Kumar et al. (2016) evaluated the interval-valued reliability of the proposed system 2-out-4 system based on state performance of elements and using universal generating function technique. Xu et al. (2016) studied a dynamic diagnosis method based on interval-valued algorithm and computed reliability and sensitivity indices of proposed updating strategy. Kozine and Krymsky (2017) computed interval-valued reliability of the considered system and used optimal central theory and also solved non-linear optimization problems.

In the above discussion, in previous years many researchers computed the reliability and others measures of SWS using UGF techniques and many more. In this paper, the interval-valued reliability of SWS in upper and lower form of the element and with the help of algorithms and obtain the reliability of the proposed system have been evaluated.

\section{Nomenclature}

$R=$ Reliability of the system.

$F=$ Unreliability of the system.

$c, d$ and $t=$ intervals numbers.

$U_{i}=$ universal generating function of element $i$.

$G_{i}=$ interval continuous sequence of state performance of $i$ element.

$k_{i}=$ state performance of $i$ element.

$p_{i}=$ state performance probability of element $i$.

$\bar{p}=$ upper probability of system.

$\underline{p}=$ lower probability of system.

$\beta_{f}=$ operator function.

$j=$ multi-state elements.

$r=$ consecutive element of the system.

\section{Definitions}

\subsection{Sliding Window System}

The SWS is a kind of consecutive $k$-out-of- $r$-from- $n$ system, consisting of $n$ ordered units and the system fails if at least $k$ out of each $r$ consecutive units fail. Each unit can have two states namely completely working or totally failed. 
International Journal of Mathematical, Engineering and Management Sciences

Vol. 4, No. 1, 108-115, 2019

https://dx.doi.org/10.33889/IJMEMS.2019.4.1-010

\subsection{Interval-Valued Based on Universal Generating Function}

The interval-valued of state performance can be defined as Pan et al. (2016)

$\left\{G_{i}^{I}\right\}=\left\{\left[G_{i, 1}^{I}\right], \ldots,\left[G_{i, k_{i}}^{I}\right], \ldots,\left[G_{i, m_{i}}^{I}\right]\right\}$, where $\left[G_{i, k_{i}}^{I}\right]=\left[\left[\underline{G}_{i, k_{i}}^{I}\right],\left[\bar{G}_{i, k_{i}}^{I}\right]\right]$ and $k_{i}$ represented the state performance of the elements in lower and upper form i.e.

$\left[\underline{G}_{i, k_{i}}\right]=\left[\underline{G}_{i, k_{i}}^{L}, \underline{G}_{i, k_{i}}^{U}\right],\left[\bar{G}_{i, k_{i}}\right]=\left[\bar{G}_{i, k_{i}}^{L}, \overline{\boldsymbol{G}}_{i, k_{i}}^{U}\right]$.

The universal generating function $U$ of interval-valued given as

$U_{i}(z)=\sum_{k_{i}}^{m_{i}}\left[p_{i, k_{i}}\right] z^{\left[\bar{G}_{i, k_{i}}\right]}$, where $i=1,2, \ldots, n, k_{i}=1,2, \ldots, m_{i}$.

\section{Some Basic Operations of Interval Arithmetic}

Jaulin et al. (2001) discussed an interval number such as $[c]=[c, \bar{c}]$, where $\bar{c} \geq \underline{c}$ and $\underline{c} \geq 0$. Suppose $c, d$ and $t$ be the interval number and operations are used as $[c]=[\underline{c}, \bar{c}]$ and $[d]=[\underline{d}, \bar{d}]$ $(\underline{c} \geq 0, \underline{d} \geq 0)$ given as

(i) $[c]+[d]=[\underline{c}+\underline{d}, \bar{c}+\bar{d}]$.

(ii) $[c]-[d]=[\underline{c}-\underline{d}, \bar{c}-\bar{d}]$.

(iii) $[c] \cdot[d]=[\underline{c d}, \overline{c d}]$ $\max \{[c],[d]\}=[\max \{\underline{c}, \underline{d}\}, \max \{\bar{c}, \bar{d}\}]$
$\min \{[c],[d]\}=[\min \{\underline{c}, \underline{d}\}, \min \{\bar{c}, \bar{d}\}]$.

(iv) Distributed law for $(\underline{c} \geq 0, \underline{d} \geq 0$, and $\underline{t} \geq 0)$

$$
[c]([d]+[t])=[c][d]+[c][t] \text {. }
$$

\section{Algorithm for Obtain the Reliability of SWS (Levitin, 2005)}

Step (i) Compute the $u$-function of the element, allocate $F=0, U_{1-r}(z)=z^{0, g_{0}}$.

Step (ii) Restate the value for $j=1,2, \ldots, k$.

Step (iii) Obtain $U_{j-r+1}(z)=U_{j-r}(z) \phi U_{j}(z)$.

Step (iv) Remove failure terms of the condition $j \geq r$ and sum the values $\beta_{f}\left(U_{j-r+1}(z)\right)$ to $F$.

Step (v) Evaluate the reliability of SWS such as $R=1-F$. 
International Journal of Mathematical, Engineering and Management Sciences

Vol. 4, No. 1, 108-115, 2019

https://dx.doi.org/10.33889/IJMEMS.2019.4.1-010

\section{Illustration}

Consider SWS with $n=4, r=2$ and $W=3$ as shown in Figure 1. and each element of the system have of two states: working or failed. Let us suppose state performance of the element from 1 to 4 are $1,2,3,4$, respectively.

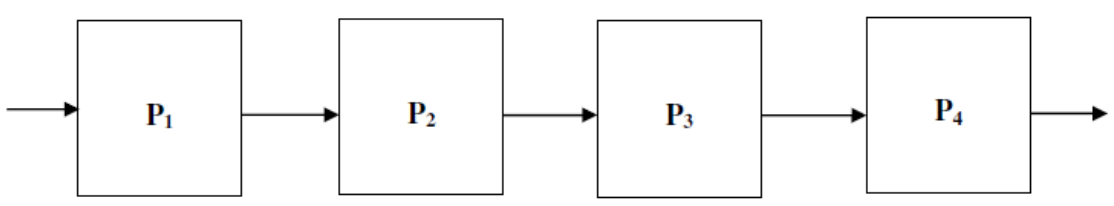

Figure 1. Block diagram of SWS with $n=4, r=2$ and $w=3$

Now, the u-function of the SWS is $U_{i j}(z)=p_{i j} z^{i}+\left(1-p_{i j}\right) z^{0}$; where, $i=j=1,2,3,4$, here $P_{i j}$ is the probability and $z^{i}$ is the performance rate and $z^{0}$ non- performance rate.

Now, the $u$-functions of the SWS in form of individual elements $(i=1,2,3,4)$ are defined

$U_{1}(z)=p_{11} z^{1}+\left(1-p_{11}\right) z^{0}$,

$U_{2}(z)=p_{21} z^{2}+\left(1-p_{21}\right) z^{0}$,

$U_{3}(z)=p_{31} z^{3}+\left(1-p_{31}\right) z^{0}$,

$U_{4}(z)=p_{41} z^{4}+\left(1-p_{41}\right) z^{0}$.

Now by the definition of interval-valued given as

$U_{11}(z)=\left(\underline{p_{11}} \overline{p_{11}}\right) z^{1}+\left(\underline{q_{11}} \overline{q_{11}} z^{1}\right) z^{0}$,

$U_{12}(z)=\left(\underline{p_{21}} \overline{p_{21}}\right) z^{2}+\left(\underline{q_{21}} \overline{q_{21}} z^{1}\right) z^{0}$,

$U_{13}(z)=\left(\underline{p_{31} \overline{p_{31}}}\right) z^{3}+\left(\underline{q_{31} \overline{q_{31}}} z^{1}\right) z^{0}$,

$U_{14}(z)=\left(\underline{p_{41}} \overline{p_{41}}\right) z^{4}+\left(\underline{q_{41}} \overline{q_{41}} z^{1}\right) z^{0}$.

From algorithm of SWS, one obtain an initial element of the $u$-function are given as For $j=1$

$$
\begin{aligned}
U_{0}(z) & =\phi\left(U_{-1}(z), U_{1}(z)\right) \\
U_{0}(z) & =\phi\left(z^{0,(0,0)}, \overline{p_{11}} \overline{p_{11}} z^{1}+\left(\underline{q_{11}} \overline{q_{11}} z^{1}\right) z^{0}\right) \\
& =\left(\underline{p_{11}} \overline{p_{11}}\right) z^{0(0,1)}+\left(\underline{q_{11}} \overline{q_{11}} z^{1}\right) z^{0(0,0)} .
\end{aligned}
$$


International Journal of Mathematical, Engineering and Management Sciences

Vol. 4, No. 1, 108-115, 2019

https://dx.doi.org/10.33889/IJMEMS.2019.4.1-010

For $j=2$

$$
\begin{aligned}
U_{1}(z)=\phi & \left(U_{0}(z), U_{2}(z)\right) \\
= & \phi\left(\left(\underline{p_{11}} \overline{p_{11}}\right) z^{0,(0,1)}+\left(\underline{q_{11}} \overline{q_{11}}\right) z^{0,(0,0)} \otimes\left(\underline{p_{21}} \overline{p_{11}}\right) z^{2}+\left(\underline{q_{21}} \overline{q_{21}}\right) z^{0}\right) \\
= & \left(\underline{p_{11} p_{21}} \overline{p_{11} p_{21}}\right) z^{0,(1,2)}+\left(\underline{p_{11} q_{21}} \overline{p_{11} q_{21}}\right) z^{0,(1,0)} \\
& \left.+\left(\underline{p_{21} q_{11}} \overline{p_{21} q_{11}}\right) z^{0,(0,2)}+\left(\underline{q_{11} q_{21}} \overline{q_{11} q_{21}}\right) z^{0,(0,0)}\right)
\end{aligned}
$$

Here, according to the algorithm 4 step (iv) of condition $j \geq w$, unreliability $F$ and $U_{1}(z)$ can be written as

$$
F=\left(\underline{p_{11} q_{21}} \overline{p_{11} q_{21}}\right)+\left(\underline{p_{21} q_{11}} \overline{p_{21} q_{11}}\right)+\left(\underline{q_{11} q_{21}} \overline{q_{11} q_{21}}\right)
$$

and

$U_{1}(z)=\left(\underline{p_{11} p_{21}} \overline{p_{11} p_{21}}\right) z^{0,(1,2)}$.

For $j=3$

$$
\begin{aligned}
U_{2}(z) & =\phi\left(U_{1}(z), U_{3}(z)\right) \\
& =\left(\left(\underline{p_{11} p_{21}} \overline{p_{11} p_{21}}\right) z^{0,(1,2)} \otimes\left(\underline{p_{31}} \overline{p_{31}}\right) z^{3}+\left(\underline{\underline{q_{31}} \overline{q_{31}}}\right) z^{0}\right) \\
& =\left(\underline{p_{11} p_{21} p_{31}} \overline{p_{11} p_{21} p_{31}}\right) z^{1,(2,3)}+\left(\underline{p_{11} p_{21} q_{31}} \overline{p_{11} p_{21} q_{31}}\right) z^{1,(2,0)}
\end{aligned}
$$

Now using the equations (2) and (3) and combining the failure terms are given as $F=\left(\underline{p_{11} p_{21} q_{31}} \overline{p_{11} p_{21} q_{31}}\right)$

For $j=4$

$$
\begin{aligned}
U_{3}(z) & =\phi\left(U_{2}(z), U_{4}(z)\right) \\
& =\left(\underline{p_{11} p_{21} p_{31}} \overline{p_{11} p_{21} p_{31}}\right) z^{1,(2,3)} \otimes\left(\left(\underline{p_{41}} \overline{p_{41}}\right) z^{4}+\left(\underline{q_{41}} \overline{q_{41}}\right) z^{0}\right) \\
& =\left(\underline{p_{11} p_{21} p_{31} p_{41}} \overline{p_{11} p_{21} p_{31}} p_{41}\right) z^{2,(3,4)}+\left(\underline{p_{11} p_{21} p_{31} q_{41}} \overline{p_{11} p_{21} p_{31}} q_{41}\right) z^{2,(3,0)}
\end{aligned}
$$

and

$$
F=\left(\underline{p_{11} q_{21}} \overline{p_{11} q_{21}}\right)+\left(\underline{p_{21} q_{11}} \overline{p_{21} q_{11}}\right)+\left(\underline{q_{11} q_{21}} \overline{q_{11} q_{21}}\right)+\left(\underline{p_{11} p_{21} q_{31}} \overline{p_{11} p_{21} q_{31}}\right) .
$$

Hence, from equations (4), reliability $\mathrm{R}$ of the system expressed as

$$
R=\left(\underline{p_{11} p_{21} p_{31} p_{41}} \overline{p_{11} p_{21} p_{31}} p_{41}\right)+\left(\underline{p_{11} p_{21} p_{31} q_{41}} \overline{p_{11} p_{21} p_{31}} q_{41}\right)
$$

Now taking internal valued and we get,

$$
\begin{aligned}
& \underline{p}_{11}=0.97, \bar{p}_{11}=0.978, \underline{p}_{21}=0.957, \bar{p}_{21}=0.962, \underline{p}_{31}=0.948, \bar{p}_{31}=0.958, \underline{p}_{41}=0.92, \\
& \bar{p}_{41}=0.935, \underline{q}_{41}=0.08, \bar{q}_{41}=0.06 .
\end{aligned}
$$


International Journal of Mathematical, Engineering and Management Sciences

Vol. 4, No. 1, 108-115, 2019

https://dx.doi.org/10.33889/IJMEMS.2019.4.1-010

Hence putting all these values in equation (5), we get the reliability of the SWS as $R=[0.88,0.8968]$.

\section{Conclusion}

Evaluation of the interval-valued reliability of any systems has many methods or techniques such as supplementary, fuzzy and Markov chain techniques etc. UGF technique gives improve results in case of interval-valued. The present work obtains interval-valued reliability in lower and upper case using UGF technique and the computed reliability of the proposed system is $R=[0.88,0.8968]$.

\section{Conflict of Interest}

The authors confirm that this article contents have no conflict of interest.

\section{Acknowledgement}

The authors would like to express their sincere thanks to the referee and for their valuable suggestions towards the improvement of the paper.

\section{References}

Chachi, J., \& Hesamian, G. (2015). Interval-valued parametric distribution functions: application to system reliability analysis. Journal of Mathematical Extension, 10, 87-105.

Destercke, S., \& Sallak, M. (2013). An extension of universal generating function in multi-state systems considering epistemic uncertainties. IEEE Transactions on Reliability, 62(2), 504-514.

Gennat, M., \& Tibken, B. (2008, April). Sensitivity analysis using interval arithmetic. In Information and Communication Technologies: From Theory to Applications, 2008. ICTTA 2008. 3rd International Conference on (pp. 1-6). IEEE.

Guo, H. X., Pang, X. B., Yang, X. F., \& Cheng, L. Z. (2013). Discrete interval-valued stress-strength interference model based on the extended universal generating function. In Applied Mechanics and Materials (Vol. 246, pp. 441-445). Trans Tech Publications.

Jaulin, L., Kieffer, M., Didrit, O., \& Walter, E. (2001). Applied interval analysis: with examples in parameter and state estimation, robust control and robotics (Vol. 1). Springer Science \& Business Media.

Kozine, I., \& Krymsky, V. (2017). Computing interval-valued reliability measures: application of optimal control methods. International Journal of General Systems, 46(2), 144-157.

Kumar, A., Singh, S. B., \& Ram, M. (2016, November). Interval-valued reliability assessment of 2-out-of-4 system. In Emerging Trends in Communication Technologies (ETCT), International Conference on (pp. 1-4). IEEE.

Levitin, G. (2001). Redundancy optimization for multi-state system with fixed resource-requirements and unreliable sources. IEEE Transactions on Reliability, 50(1), 52-59.

Levitin, G. (2002). Optimal allocation of elements in a linear multi-state sliding window system. Reliability Engineering \& System Safety, 76(3), 245-254. 
International Journal of Mathematical, Engineering and Management Sciences

Vol. 4, No. 1, 108-115, 2019

https://dx.doi.org/10.33889/IJMEMS.2019.4.1-010

Levitin, G. (2003a). Common supply failures in linear multi-state sliding window systems. Reliability Engineering \& System Safety, 82(1), 55-62.

Levitin, G. (2003b). Linear multi-state sliding-window systems. IEEE Transactions on Reliability, 52(2), 263-269.

Levitin, G. (2005). The universal generating function in reliability analysis and optimization (p. 442). London: Springer.

Li, C. Y., Chen, X., Yi, X. S., \& Tao, J. Y. (2011). Interval-valued reliability analysis of multi-state systems. IEEE Transactions on Reliability, 60(1), 323-330.

Ram, M. (2013). On system reliability approaches: a brief survey. International Journal of System Assurance Engineering and Management, 4(2), 101-117.

Ram, M., \& Singh, S. B. (2010). Availability, MTTF and cost analysis of complex system under preemptive-repeat repair discipline using Gumbel-Hougaard family copula. International Journal of Quality \& Reliability Management, 27(5), 576-595.

Ushakov, I. (1986). Universal generating function, Journal of Computer and System Sciences, 24, 118-129.

Ushakov, I. A. (Ed.). (1994). Handbook of reliability engineering. John Wiley \& Sons.

Utkin, L. V., \& Kozine, I. O. (2005). Computing system reliability given interval-valued characteristics of the elements. Reliable Computing, 11(1), 19-34.

Wang, W., Xiong, J., \& Xie, M. (2016). A study of interval analysis for cold-standby system reliability optimization under parameter uncertainty. Computers \& Industrial Engineering, 97, 93-100.

Xu, X., Zhang, Z., Xu, D., \& Chen, Y. (2016). Interval-valued evidence updating with reliability and sensitivity analysis for fault diagnosis. International Journal of Computational Intelligence Systems, 9(3), 396-415. 